\title{
Force-Based Calibration of a Particle System for Realistic Simulation of Nonlinear and Viscoelastic Soft Tissue Behavior
}

\author{
Bektas Baybora Baran and Cagatay Basdogan \\ College of Engineering, Koc University, Istanbul, Turkey, 34450 \\ \{bekbaran, cbasdogan\}@ku. edu.tr
}

\begin{abstract}
We present a new approach for realistic visio-haptic simulation of nonlinear and viscoelastic behavior of an organ tissue using a particle model. The spring and damper coefficients of the particle model are calibrated using a derivative-free optimization technique such that its behavior mimics the behavior of a corresponding finite element (FE) model. In our approach, we first conduct static indentation and stress relaxation experiments on the FE model to record a) force versus displacement and b) force versus time responses of the surface nodes, respectively. We then use these data sets to calibrate the spring and damper coefficients of the particle model such that its force response is similar to that of the FE model. To test the feasibility of our approach, we compare the static and dynamic behavior of the particle model to that of the FE model under the influence of gravity.
\end{abstract}

Keywords: Viscoelasticity, soft tissue behavior, surgical simulation, particle system.

\section{Introduction}

The particle system approach is a widely used mesh-free method in simulating soft tissue behavior [1]. The coefficients of springs and dampers in a particle system must be tuned individually to accurately mimic the response of the corresponding physical system. However, the integration of measured material properties of a soft tissue into a particle system is a highly challenging task.

Only a few studies in the past attacked the problem of parameter identification for a particle system. In [2], the authors establish a link between discrete mass-spring models and the classical theory of elasticity. In [3], a genetic optimization algorithm is used to determine the topology of the mass-spring system. In [4], a 2D mass-springdamper system is trained to behave like a nonlinear FEM. A more advanced approach is taken in [5]. By using a layer of 2D linear springs and dashpots supported by nonlinear springs in parallel, the dynamical deformations of the human thigh is simulated. In [6], a method is introduced to derive analytical expressions for the spring coefficients of a particle model based on a reference FE model.

This paper presents a novel approach for the realistic particle-based simulation of hyper-viscoelastic organ behavior. Most of the earlier studies in this area have focused

A.M.L. Kappers et al. (Eds.): EuroHaptics 2010, LNCS 6191, Part I, pp. 23-28, 2010.

(C) Springer-Verlag Berlin Heidelberg 2010 
on matching the visual response of a particle model to that of the corresponding FE model, but not the strain- and time-dependent force responses. In our approach, we first perform characterization experiments with a FE model of an organ mesh in virtual domain: strain- and time-dependent force responses of a set of nodes selected on the surface are measured through static indentation and stress relaxation experiments, respectively. Then, a particle model is constructed from the same geometric mesh. Each particle in our model is connected to its neighbors by a Maxwell Solid (MS), made of 3 springs and 2 dampers $(\mathrm{N}=2)$. Finally, the same characterization experiments are performed on the particle model to estimate the spring and damper coefficients of each MS via a set of novel optimization algorithms such that the haptic response of the particle model successfully imitates that of the FE model.

\section{Numerical Model of the Particle System}

To model the viscoelastic relaxation behavior of a soft tissue, the most basic force model is the Standard Linear Solid (SLS). The SLS is a special case of Generalized MS model when only one Maxwell arm (a spring and a damper in series) is used. In our studies, we have observed that SLS is still insufficient to model the relaxation response of soft tissues [7]. This is due to the fact that the measured force relaxation response of soft tissues is typically governed by multiple exponential decaying functions having different time constants and a single time constant is not capable of modeling the actual response. For this reason, we use a MS model with two Maxwell arms $(\mathrm{N}=2)$ (Fig. 1).

In addition to modeling the viscoelastic force response, we modify the original MS model to simulate the strain-dependent force response of soft tissues. This is achieved by replacing the linear spring responsible from the static response in the original MS model with a strain-dependent nonlinear spring. Hence, the spring coefficient $K_{\infty}$ in the MS model (Fig. 1) is written as a function of strain as:

$$
K_{\infty}(\varepsilon)=K_{0}+a \cdot \varepsilon+b \cdot \varepsilon^{2}
$$

This representation enables us to model the strain-dependent nonlinear material properties of the organ.

a)

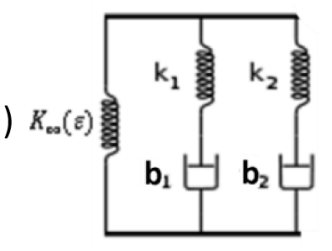

b)

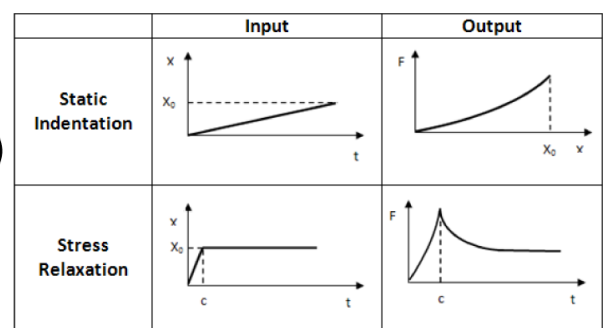

Fig. 1. a) Maxwell Solid with two Maxwell arms, b) The force response of the Maxwell solid model to static and ramp and hold stimuli 
To calculate the force response of particles in our particle model in real-time, Verlet time integration method is chosen, which is explicit and conservative in the long run. We use the following scheme for the calculation of the forces on each connector joining two particles to each other via the MS model $(\mathrm{N}=2)$ :

$$
\begin{gathered}
F^{t}=K_{\infty}(\varepsilon) \cdot \Delta x^{t}+\sum_{i=1}^{2} F_{\text {maxwell_arm } \_i}{ }^{t} \\
F_{\text {maxwell_arm_ } i}{ }^{t}=\frac{k_{i} \cdot b_{i}}{k_{i} \cdot \Delta t+b_{i}}\left(\left(\Delta x^{t}-\Delta x^{t-1}\right)+F_{\text {maxwell_arm } \_}{ }^{t-1} / k_{i}\right)
\end{gathered}
$$

Here $\Delta x^{t}$ is the difference between the current connector length and its original length, and $\Delta t$ is the time step used for the integration.

\section{Calibration of the Particle System}

We construct our deformable FE model in this study by inputting the Mooney-Rivlin parameters for hyperelasticity and the Prony parameters for viscoelasticity to ANSYS, which were obtained from the experiments performed on pig liver [7]. In order to calibrate the particle model such that its haptic response resembles to the FE model, we first identify a set of test points on the surface of the geometric mesh used for both models. We then perform static indentation and stress relaxation experiments with the FE model using these test points in ANSYS and record their a) force versus displacement and $b$ ) force versus time responses.

The calibration of the springs and dampers of the particle system is achieved in two sequential optimization steps using the recorded force data. In the first step, a unique set of parameters for all connectors (i.e. $K_{0}, a, b, k_{1}, b_{1}, k_{2}, b_{2}$ ), is determined by considering the force-responses of all test points in the optimization. This step is called global tuning and helpful to determine the initial values of parameters. In the second step, starting from the initial values determined in the first step, we fine-tune the parameters of each connector by considering the individual response of its nodes. This step is called local tuning. In both steps, the optimization of the parameters is achieved using Nelder-Mead derivative-free optimization method.

More specifically, the nonlinear strain-dependent material parameters of our particle model for each connector (i.e. $K_{0}, a, b$ ), are obtained from the force versus displacement responses of the test points under the static loading (Fig. 1). Since the indentation rate during a static loading is very slow, all time-dependent effects contributing to the force response are negligible. Hence, the force response during static loading is governed by the strain-dependent nonlinear spring $K_{\infty}(\varepsilon)$ only. An analogy is possible for the stress relaxation test and hence the related material parameters are $k_{1}, b_{1}, k_{2}, b_{2}$. The viscoelastic force relaxation response of each test point is governed by the two time constants in our MS model $\left(\tau_{1}=b_{1} / k_{1}, \tau_{2}=b_{2} / k_{2}\right)$. Hence, the hyperelastic material parameters $K_{0}, a, b$ are optimized first using the recorded static indentation data and then the viscoelastic material parameters $k_{1}, b_{1}, k_{2}, b_{2}$ are optimized using the recorded stress relaxation data. To optimize the parameters of the particle model, a cost function is defined as: 


$$
G_{\text {cost } \_j}=\sum_{i=1}^{N}\left(F_{\text {ref } \_i-} F_{\text {sim } \_}\right)^{2}{ }^{2}, G_{\text {Total cost }}=\sum_{j=1}^{M} G_{\text {cost } \_j}
$$

Here, $F_{\text {sim }}$ represents the force response generated by the particle model and $F_{\text {ref }}$; represents the reference force response obtained from the FE simulations performed in ANSYS. $N$ is the number of data samples taken from the response curves, and $\mathrm{M}$ is the number of test points used for the optimization.

The goal of the optimization process is to find a single set of material parameters that minimize $G_{\text {Total cost }}$. However, we observe that it is not possible to obtain a set of optimum material parameters that satisfy the individual force responses of all the test points in one-step optimization. For this reason, in the global tuning step, all the connectors are assumed to have the same values of material parameters and an initial set is obtained. In the local tuning step, using this data set as the initial guess, we further improve the quality of the fit by fine-tuning the material parameters of each connector. The optimization is performed locally by modifying only the connectors that are in the first degree neighborhood of the test points.

\section{Results}

To demonstrate our approach, we compared the static and dynamic behavior of a particle model of a liver mesh to that of the corresponding FE model under the influence of gravity. The liver mesh consists of 887 tetrahedral elements, 269 nodes, and 1358 connectors. A total of 31 test points on the mesh surface were used to calibrate the particle model. The test points were chosen such that all the connectors on the mesh surface were considered in the optimization calculations. It took about 8 hours to complete the calibration process in a dual-core Pentium PC, each having $3 \mathrm{GHz}$ CPU. In figures $2 \mathrm{a}$ and $2 \mathrm{~b}$, the results of the global and the local optimizations are shown for a surface node under static loading (i.e. static compression test) and ramp and hold loading (i.e. stress relaxation test). In both cases, the global tuning returns a nearby solution and then the local tuning increases the quality of the fit by locally modifying the parameters of individual connectors. The convergent behavior of the cost function as a function of the increasing number of optimization iterations is shown in figures $2 \mathrm{c}$ and $2 \mathrm{~d}$ for the global tuning step. To give an idea about the variation in the material parameters of connectors as a result of the two-steps optimization process, the values of $K_{0}$ and the time constant $\tau_{1}$ are plotted for each connector in figures $2 \mathrm{e}$ and $2 \mathrm{f}$.

The static and dynamic responses of the particle model under the influence gravity were compared to that of the FE model. For this purpose, some of the nodes at the bottom surface of the liver mesh were fixed (zero displacement) and the free nodes of the liver were allowed to move under the influence of gravity (Fig. 3). The comparison for the static response is given in Fig. 3c. For the comparison of dynamic response, the surface node that is on the far right side of the liver mesh was selected (Fig. 3d) and changes in its displacement with respect to time were compared (Fig. 3e). 

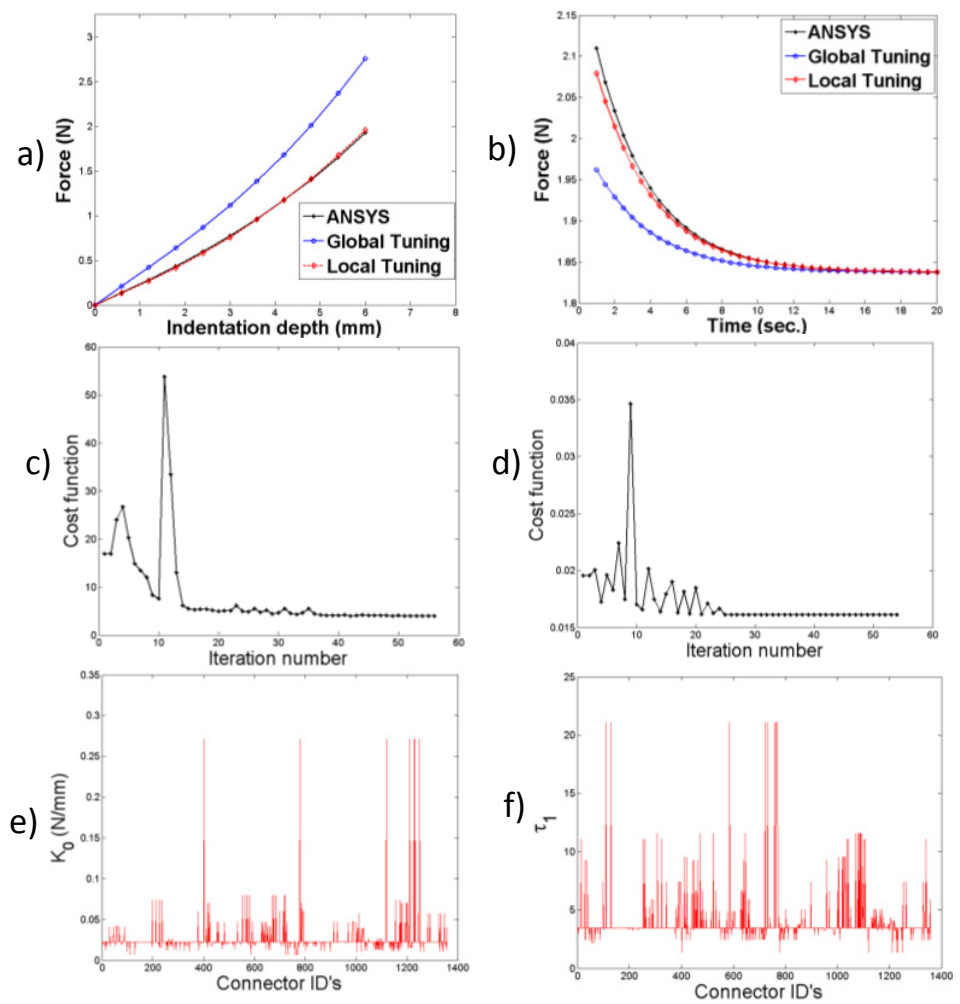

Fig. 2. The results of the global and the local optimizations for a surface node under a) static loading and b) ramp and hold loading. The evolution of the cost function during the global optimization for $\mathrm{c}$ ) the static indentation and d) the stress relaxation tests. The variations in e) the nonlinear spring coefficient $K_{0}$ in static indentation and f) the time constant $\left(\tau_{1}=b_{1} / k_{1}\right)$ in stress relaxation over the connectors.

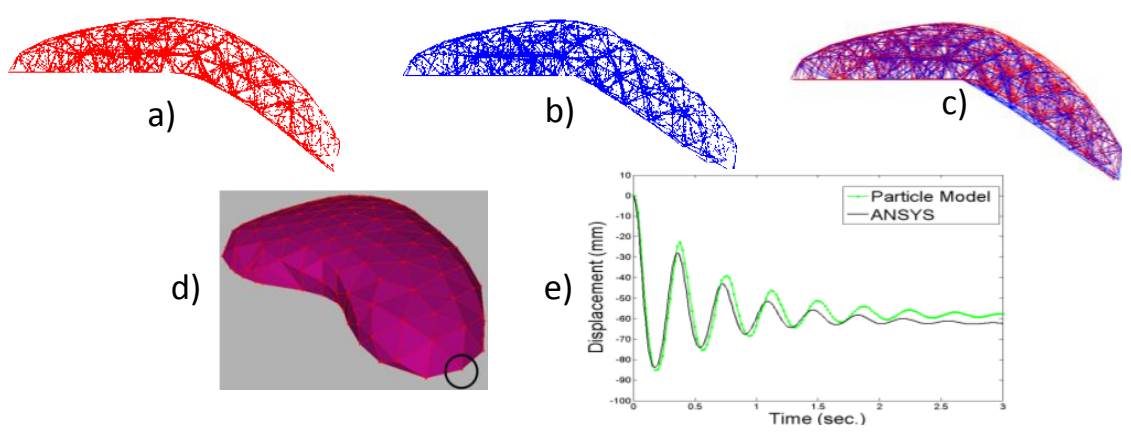

Fig. 3. a) The comparison of the static responses for the gravity test: a) the solution obtained from ANSYS, b) the solution obtained from the calibrated particle model, and c) both solutions in the same view. d) The node chosen for the comparison of dynamic response, e) The dynamic displacement of the chosen node under the influence of gravity (note that the dynamic simulation shown in the graph for 3 seconds is executed by the particle model in 1.3 seconds). 


\section{Conclusion}

A parameter estimation technique for a particle system that simulates the complex nonlinear and viscoelastic behavior of a soft organ tissue was presented. The earlier studies on parameter estimation of particle based systems are limited to the estimation of linear or nonlinear material coefficients to simulate the static response and not the time-dependent viscoelastic response. Here, it is important to emphasize that the popular Voigt connector (a spring and a damper in parallel) is not capable of modeling the "true" viscoelastic "relaxation" response of a soft tissue though it is frequently used in particle models developed to simulate the behavior of deformable objects in computer graphics. Also, the earlier studies have mostly focused on matching the visual response of a particle model to that of the reference FEM model. In our approach, we focused on matching the hyper-viscoelastic force response. Since our calibration is based on the force response rather than the visual one, the developed particle model can be more easily integrated with a haptic device for displaying realistic force feedback to a user during the simulation of surgical procedures.

\section{Acknowledgements}

The Scientific and Technological Research Council of Turkey supported this work under the contract MAG-104M283.

\section{References}

1. Basdogan, C., Sedef, M., Harders, M., Wesarg, S.: Virtual Reality Supported Simulators for Training in Minimally Invasive Surgery. IEEE Computer Graphics and Applications 27(2), 54-66 (2007)

2. Etzmuss, O., Gross, J., Strasser, W.: Deriving a Particle System from Continuum Mechanics for the Animation of Deformable Objects. IEEE Transactions on Visualization and Computer Graphics 9(4), 538-550 (2003)

3. Bianchi, G., Harders, M., Székely, G.: Mesh topology identification for mass-spring models. In: Ellis, R.E., Peters, T.M. (eds.) MICCAI 2003. LNCS, vol. 2878, pp. 50-58. Springer, Heidelberg (2003)

4. Pezzementi, Z., Ursu, D., Misra, S., Okamura, A.M.: Modeling Realistic Tool-Tissue Interactions with Haptic Feedback: A Learning-Based Method. In: 16th Symposium on Haptic Interfaces for Virtual Environments and Teleoperator Systems, Reno, pp. 209-215 (2008)

5. d'Aulignac, D., Cavusoglu, M.C., Laugier, C.: Modeling the Dynamics of the Human Thigh for a Realistic Echographic Simulator with Force Feedback. In: Taylor, C., Colchester, A. (eds.) MICCAI 1999. LNCS, vol. 1679, pp. 1191-1198. Springer, Heidelberg (1999)

6. Lloyd, B.A., Székely, G., Harders, M.: Identification of Spring Parameters for Deformable Object Simulation. IEEE Transactions on Visualization and Computer Graphics 13(5), 1081-1094 (2007)

7. Samur, E., Sedef, M., Basdogan, C., Avtan, L., Duzgun, O.: A Robotic Indenter for Minimally Invasive Measurement and Characterization of Soft Tissue Behavior. Medical Image Analysis 11(4), 361-373 (2007) 